\title{
Nutrition and Dry Eye
}

\author{
Pasquale Aragona $\cdot$ Laura Rania • \\ Antonio Micali · Domenico Puzzolo
}

Published online: 15 March 2013

(C) Springer Science + Business Media New York 2013

\begin{abstract}
The tear film is a complex mixture of molecules such as lipids, mucins and water, whose function is to protect the eye from external factors such as environmental stimuli, traumas and infections. It is secreted by specific structures, but also, in part, derives from the blood stream. Substances taken with food can directly or indirectly influence tear film composition so inducing changes of the ocular surface. When the tear film is altered a condition known as dry eye may develop. Diet derived substances can be of help in the treatment of this condition. Among these substances essential fatty acids, of the omega- 6 and 3 series, can play a role in the control of inflammation and in promoting the recovery of the ocular surface structures. Also some aminoacid implementation, with molecules that participate to collage composition, may participate in the recovery of the ocular surface structures undergoing the inflammatory stress.
\end{abstract}

Keywords Nutrition · Dry eye · Sjögren's syndrome · Ocular surface $\cdot$ Corneal epithelium $\cdot$ Conjunctival

P. Aragona $(\bowtie) \cdot$ L. Rania

Ocular Surface Diseases Unit, Department of Experimental

Medical-Surgical Sciences, University of Messina, Policlinico

Universitario, Via Consolare Valeria 1, I-98125 Messina, Italy

e-mail: paragona@unime.it

L. Rania

e-mail: laurarania@libero.it

\section{A. Micali · D. Puzzolo}

Department of Biomedical Sciences and Morphofunctional Imaging, University of Messina, Policlinico Universitario, Via Consolare Valeria 1, I-98125 Messina, Italy

e-mail: amicali@unime.it

D. Puzzolo

e-mail: puzzolo@unime.it epithelium · Omega-3 - Omega-6 · Vitamins · Diabetes · Resolvins $\cdot$ Neuroprotectins $\cdot$ Amino acids

\section{Introduction}

Tear film is a complex structure, whose composition depends on the activity of several secretory elements, each contributing to the production of components characterized by a peculiar chemical structure, which accounts for its adequate stability on the ocular surface.

The main tear film components are lipids, water with electrolytes and proteins and glycoproteins, each one of these being secreted by specific structures, but also, in part, deriving from the blood stream. While it is clear that the meibomian gland-derived lipid layer is distributed on the surface of the aqueous layer, thus forming the outermost tear film lipid layer (TFLL), the aqueous tears are a mixture of lacrimal fluid and mucins, without a distinct mucin layer [1].

The TFLL is produced by the meibomian glands and consists of a complex mixture of various polar and nonpolar lipids containing cholesteryl esters, triacylglycerol, free cholesterol, free fatty acids, phospholipids, wax esters and diesters. The TFLL is usually described as a two-layered structure: polar lipids form the lower sublayer and nonpolar lipids form the upper portion, that is in contact with the air. It is likely that lipids are not the only class of molecules participating in TFLL structure. In fact, intercalated proteins were also demonstrated, which are now considered an intrinsic part of TFLL [2•]. Many proteins spontaneously populate the air-liquid interface; because of their surface-active attitude, they tend to create a surface protein layer, which will contribute to lowering the surface tension of water. These proteins translocate from the bulk aqueous phase towards the surface, where they undergo 
denaturation characterized by irreversible conformational changes, typically unfolding, that prevent proteins from submerging back into the depth of the aqueous layer. This results in the formation of a protein layer interposed between the aqueous and lipid layers [2•].

The aqueous layer is primarily originated by the lacrimal gland secretion. Also contributing are the secretions of the accessory glands of Krause, placed in the fornices, and those of Wolfring, found in the upper and lower tarsi. Extravasation of material from conjunctival capillaries and intra- and intercellular components of the conjunctiva and cornea contribute part of the aqueous layer [3]. Therefore, it is composed of water, inorganic salts, proteins and enzymes, including amylase, lysozyme, lactoferrin and tear-specific prealbumin, glycoproteins [4], and other organic compounds, such as glucose and urea.

The interface between the aqueous layer and the epithelial cell membrane is characterized by the presence of highly glycosylated glycoproteins, produced by both the lacrimal glands and the corneo-conjunctival epithelial cells, which form the glycocalyx. This is composed for up to the $80 \%$ of carbohydrates and emanates from the tips of the microplicae, extending up to $500 \mathrm{~nm}$ from the cellular membrane [1,5]. Sequencing of mucin genes has led to the identification of two categories of mucins: secreted and membrane associated, either in a spanning or in a tethered manner.

Dry eye syndrome (DES) is the consequence of tear film changes that can arise from aqueous deficiencies (Aqueous deficient dry eye-ADDE) or increased evaporation rate (Evaporative dry eye-EDE), the latter mainly due to lipids alterations. Both these conditions will result in increased concentration of tear film solutes and, therefore, in tear hyperosmolarity [6]. The inflammation resulting from this condition plays a fundamental role in DE pathogenesis, as it is both the consequence and the mechanism responsible for the maintenance of the ocular surface damage due to tear impairment.

Nutrition and nutritional status can produce relevant effects on the ocular surface function.

\section{Detrimental Effects of Nutrition and Nutritional Status}

Nutrients may be detrimental, for example in the case of complex, saturated lipids, which may induce changes of the lipid composition of the tear film. In the case of high levels of serum cholesterol, there could be significant sequelae in meibomian secretion $[3,7 \bullet]$. In fact, the excess of cholesterol in the meibum would increase its melting point determining the production of a semi-solid secretion with a reduction of lipids delivery into the tear film and consequent hyperevaporation, possibly resulting in a dysfunctional tear syndrome.

Furthermore, water soluble products could be delivered in the aqueous phase, changing its chemico-physical properties and determining an increased tear osmolarity and film instability. This can be the case of glucose in diabetic patients [8].

Also the nutritional status can affect the ocular surface. This is the case of vitamin deficiencies, among which vitamin A is particularly relevant because of its role in ocular surface epithelium trophism. Vitamin A deficiency has been well described in different areas of the world in people with low socio-economic status [9]. In these subjects the ocular surface presents characteristic alterations consisting in diffuse keratinization with relevant squamous metaplasia of the corneo-conjunctival epithelium, which may lead to severe impairment of the quality of vision. This condition can be reversed by the systemic administration of vitamin A or beta carotene (provitamin A). This deficient condition is also present in western countries, as a consequence of malabsorption due to bariatric surgery in obese patients [10] or in intestinal disorders, such as coeliac disease [11], and in malnutrition due to severe anorexia nervosa $[12,13]$; it can be reversed with an appropriate therapy (Fig. 1).

The water soluble vitamin pyridoxine (vitamin B6) was shown to have an effect on the immune response. Deficiency of this vitamin induced atrophy of lymphoid organs, with consequent reduced lymphocyte production, altered antibody response and interleukin 2 production [14].
Fig. 1 a Clinical picture of corneal keratinisation induced by vitamin A deprivation in a 16 years old female patient with nervous anoressia. b The same eye after 2 months of treatment with appropriate vitamin A administration
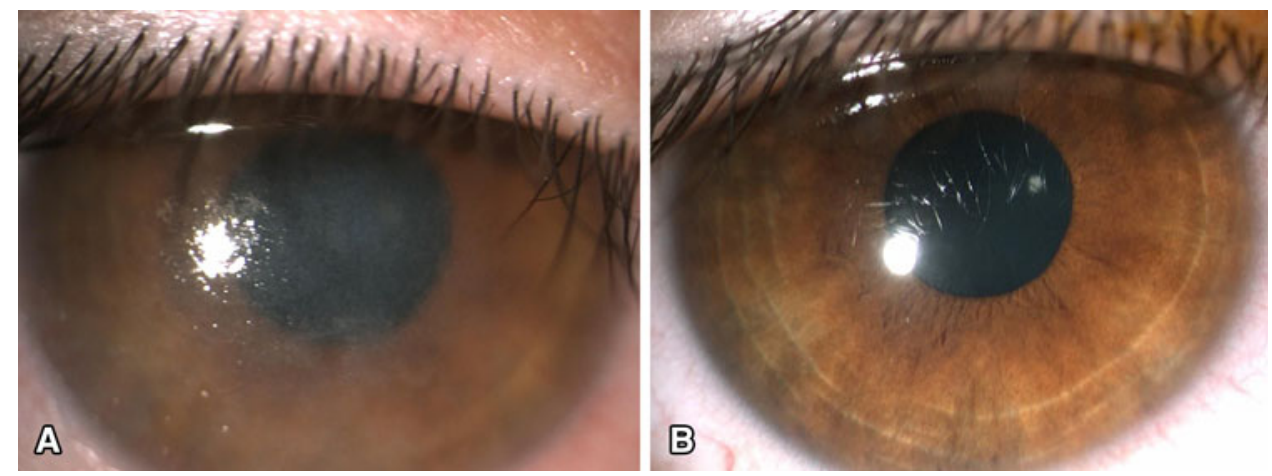
Deficiencies of oligoelements, such as $\mathrm{Zn}, \mathrm{Cu}, \mathrm{Mn}$, and of vitamins $\mathrm{C}$ and $\mathrm{E}$ were able to determine a loss of goblet cells in the conjunctiva and a significant decrease of microvilli and microplicae in the superficial cells of the conjunctiva and cornea [15].

\section{Favorable Effects of Nutrition and Nutritional Status}

Nutrients can be used to obtain favorable, therapeutic effects on the ocular surface. Among the several substances for which a favorable effect has been described, of particular relevance seems to be the role of poly-unsaturated essential fatty acids (PUFAs) for their anti inflammatory, regenerating and neuroprotective effects, and amino acids (AA) for their possible positive role on the corneal structure.

\section{PUFAs}

\section{PUFAs and Ocular Surface}

Omega-3 and -6 are PUFAs, also termed "essential," because they cannot be synthesized by the human body, due to the lack of the necessary enzymes, and must be introduced with the diet. Essential fatty acids contain a carboxyl group $(\mathrm{COOH})$ at one end, and so are among the group of compounds known as carboxylic acids. The final position at the end of the chain opposite the carboxyl group is known as $\omega$ (omega, the final letter of the Greek alphabet) and the terms omega- 3 and -6 refer to the location of the first double-bond in relation to the $\omega$ end of the chain $[16 \bullet \bullet]$.

The PUFAs can be taken with food, as they are widely distributed in vegetables and animals [17]. In particular, omega-3 are found in fish and fish oils, and in green leafy vegetables, such as broccoli and spinach, in nuts and beans, in seeds and oils. Omega-6 are available in many widespread oils, such as those derived from corn, peanut, safflower, rapeseed, sunflower; other common sources of omega- 6 are poultry, eggs, cereals and whole-grain breads [18]. It must be considered that the limited amounts of omega-3 in meats become nutritionally important considering the large quantities of beef, pork, poultry present in Western diets [19].

Among people who consume typical Western diets, the average intake of omega- 3 fatty acids, found in fish, is about $150 \mathrm{mg}$ per day. This is equivalent to consuming about one fish meal every 10 days, which is below the combined intake of dietary eicosapentaenoic and docosahexaenoic acids $(650 \mathrm{mg} / \mathrm{d})$ recommended at a workshop held in 1999 at the US National Institutes of Health.
Similarly, the International Society for the Study of Fatty Acids and Lipids recommends an intake of at least $500 \mathrm{mg}$ daily. The American Heart Association suggests that people without coronary heart disease have two fish meals each week (at least $300 \mathrm{mg}$ of omega-3 fatty acids daily), whilst patients with coronary heart disease receive $1,000 \mathrm{mg}$ per day. In addition, the US Food and Drug Administration recommends that the average daily intake of omega-3 fatty acids from fish should not exceed $3,000 \mathrm{mg}$ because of possible adverse effects related to glycemic control, increased bleeding tendencies and elevations in low-density lipoprotein cholesterol [19].

For omega-6, it was suggested one have a daily adequate intake, for adults, of $4.44 \mathrm{~g} /$ day for a diet of 2,000 kcal [20]. It was also suggested that higher intake could be detrimental since they are also precursors of pro inflammatory eicosanoids. However this hypothesis is based on minimal evidence, and in humans, higher intakes of omega- 6 fatty acids was not associated with elevated levels of inflammatory markers [21]. Furthrmore, it was indicated a specific ratio between omega- 6 and -3 should be maintained in order to obtain a positive effect on the organism; in fact, a dietary ratio of omega- 6 to omega- 3 fatty acids greater than 15:1 was associated with a 2.5-fold increased risk of DE [22].

\section{Physiological Role of PUFAs on the Ocular Surface}

Omega-6 PUFAs play a crucial role in brain function, as well as normal growth and development. Furthermore, they help stimulate skin and hair growth, maintain bone health, regulate metabolism, and maintain the reproductive system. Gamma-linolenic acid (GLA), a molecule synthesized from the linoleic acid into the body, plays an anti inflammatory action, as it promotes the production of prostaglandin $\mathrm{E}_{1}$ through the conversion into dihomo-gammalinolenic acid (DGLA). The anti inflammatory activity of GLA and DGLA was demonstrated on the ocular surface in patients with Sjögren's Syndrome (SS) dry eye [23]. In addition, both GLA and DGLA modulate the immune responses by acting directly on $\mathrm{T}$ lymphocytes. It has been shown that GLA reduces the autoinduction of IL-1 $\beta$ by inducing a protein that reduces pro-IL-1 $\beta$ mRNA stability. The IL- $1 \beta$ is important in host defense, but the autoinduced amplification mechanism may be excessive in genetically predisposed patients. Thus, reduction of IL-1 $\beta$ autoinduction may be protective in some patients with diseases characterized by chronic inflammation [24]. In patients with SS, the plasma and erythrocyte membrane levels of fatty acids of both the omega- 6 and -3 series of fatty acids correlates inversely with levels of $\operatorname{IgM}$ rheumatoid factor and anti SSA/Ro antibodies [25]. 
The most commonly studied omega-3 PUFAs include $\alpha$-linolenic acid (ALA), which is the precursor to eicosapentaenoic acid (EPA), which then gives rise to docosahexaenoic acid (DHA). In general, the $n-3$ pathway produces eicosanoids with anti-inflammatory and anti-aggregatory properties. They play their role either in vivo [26] or in vitro [27] through the production of anti-inflammatory eicosanoids, such as $\mathrm{PGE}_{3}$ and novel molecules that act in the termination of inflammation and the return of tissues to a state of homeostasis. A main role, among these, is played by the resolution-phase interaction products, or resolvins, which are synthesized from EPA (resolvins E) and DHA (resolvins D) and are also produced by the COX-2 in the presence of aspirin. Like eicosanoids, they are locally acting hormones. Resolvins play a role in tissues as diverse as the liver, lung, and eye. They inhibit the production of inflammatory mediators, leukocyte infiltration and cytokine expression, so reducing the time course and the scale of inflammatory processes [28].

Along with resolvins, the omega-3 fatty acids may originate other substances called neuroprotectins.

The resolvins and neuroprotectins are distinctive and highly stereospecific lipids, which are endogenous local mediators with strong anti-inflammatory effects in addition to some immunoregulatory activities at very low concentrations. They contribute to the removal of inflammatory cells and restoration of tissue integrity at the end of the inflammatory response, so participating in the resolution of inflammation. Their effect is enhanced by aspirin, which facilitates the conversion of EPA and DHA to E and D resolvins, providing a potent anti-inflammatory effect mediated by specific receptors.

The neuroprotectin D1 has anti-inflammatory effects, protects neural tissues in experimental models of stroke [29] and Alzheimer's disease [30] and promotes nerve regeneration in dry eye models and after refractive surgery [31•]. Furthermore, it promotes apoptosis of $\mathrm{T}$ cells, showing beneficial effects in inflammatory disorders such as asthma and dry eye.

\section{Therapeutic Role of PUFAs on the Ocular Surface}

As to our knowledge, there are six published clinical studies on essential fatty acids in patients with DES [23, $26,32-35 \cdot 0$ ] and, of those, only two studies report the impact of the combination of omega- 3 and omega- 6 oral supplementation in DES [33, 35••].

\section{Omega-6 Supplementation}

Systemic treatment with a total daily dose of $57 \mathrm{mg}$ of LA and $30 \mathrm{mg}$ of GLA was able to decrease chronic inflammation due to keratoconjunctivitis sicca [32], as demonstrated by the reduced expression of HLA-DR on impression cytology specimens after 45 days of treatment. Furthermore, an improvement of ocular discomfort symptoms and lissamine green ocular surface staining was also observed.

In another study [23], it was demonstrated that the oral administration of a total daily dose of $224 \mathrm{mg}$ of LA and $30 \mathrm{mg}$ of GLA was able to increase the tear levels of $\mathrm{PGE}_{1}$, an eicosanoid with known anti inflammatory properties, after 1 month of treatment. Furthermore, symptoms of dry eye and corneal fluorescein staining were most improved at the end of therapy. Fifteen days after treatment interruption, the $\mathrm{PGE}_{1}$, levels were reduced towards baseline levels as also the symptoms significantly worsened, while the corneal fluorescein staining remained improved if compared to baseline values.

In a study about dry eye in contact lens wearers [34], the oral supplementation with omega- 6 fatty acids, with a total daily dose of $300 \mathrm{mg}$ of GLA, was able to induce an overall improvement of comfort in contact lens wear after 6 months of treatment, whilst the dryness symptoms were improved after 3 months and maintained after 6 months. The tear meniscus height was also significantly increased after six months.

\section{Omega-3 Supplementation}

The omega-3 supplementation with a total daily dose of $\sim 3.3 \mathrm{~g}$ of ALA per day produced an improvement in subjects with meibomian gland dysfunction and blepharitis [26]. This treatment was able to determine a significant systemic change in plasma or red blood cells' omega- 3 level, with a reduction of the omega-6:omega- 3 ratio by the $36 \%$ in the plasma and the $31 \%$ in red blood cells. Ocular surface disease index, tear breakup time and meibum score were significantly ameliorated with treatment.

\section{Supplementation with combined Omega-3} and Omega-6

The oral supplementation with a total daily dose of $392 \mathrm{mg}$ of DHA, $28 \mathrm{mg}$ of EPA, $82 \mathrm{mg}$ of GLA, and $126 \mathrm{mg}$ of LA [33] was able to induce a not significant improvement of signs, such as Schirmer test, tear breakup time, and fluorescein and lissamine staining, after 6 months of treatment.

Another study [35••] demonstrated that a total daily dose of $427.5 \mathrm{mg}$ EPA, $285 \mathrm{mg}$ DHA, and $15 \mathrm{mg}$ GLA for 3 months induced a reduced percentage of HLA-DR-positive cells and their fluorescence intensity. A trend for improvement in symptoms and corneal staining was also noted.

\section{Aminoacids}

Aminoacids and Ocular Surface

The oral administration of substances, such as amino acids (AA), able to provide a metabolic sustain to both the 
corneo-conjunctival epithelium and the deeper structures, such as the basement membrane and the stroma, can be of help in the recovery of the ocular surface under stress conditions. In fact, the health of the stroma underlying the epithelium is essential to guarantee the epithelial integrity [36••]. A mixture of four AA (proline, lysine, glycine and leucine), which are the main components of collagen fibers in the stroma and basement membrane, was proposed to be of great advantage to the integrity of the epithelial layer [37].

Physiological Role of AA on the Ocular Surface

A mixture of AA was suggested to be an ideal diet supplementation to improve ocular surface conditions. A study highlighted the capacity of AA to preserve a good viability and functional activity of cultured conjunctival epithelial cells and a slight increase in MUC-5AC glycoprotein production [37]. In particular, AA such as glycine, proline, lysine and leucine, play a role in the processes of collagen biosynthesis [38] and epithelial remodeling in the course of wound healing [39]. Furthermore, they participate in the normal human collagen composition [40]. Glycine is a nonessential AA, which acts as a precursor of proteins [41]; the collagen molecule contains one-third glycine, as every third AA is a glycine molecule, according to the sequence GLY-X-Y [40]. Proline is also a non-essential AA in humans [42] and it plays important roles in metabolism, wound healing, and protein synthesis [43]. Together with its derivative hydroxyproline, it frequently occupies the $\mathrm{X}$ or $\mathrm{Y}$ position of the collagen sequence and constitutes about the $23 \%$ of the AA content of the collagen molecule [40]. Lysine is an essential AA in humans and it is necessary for building up all proteins in the body. In particular, during collagen biosynthesis, specific lysine residues are hydroxylated to form hydroxylysine: outside the cell, lysine and hydroxylysine residues can be oxidatively deaminated to form covalent intra- and inter-molecular cross-links between tropocollagen molecules [44]. Leucine is the main component of proteoglycans called small leucine-rich proteoglycans (SLRPs), which are among the main components of corneal stroma. The SLRPs take part to the regulation of collagen fibrillogenesis, extracellular matrix assemblage, and cell-cycle progression; therefore, they are essential to the maintaining of corneal transparency. Also, they antagonize transforming growth factor- $\beta$ induced myofibroblast transformation and fibrosis development in the cornea [45]. Furthermore, a dramatic increase of leucine incorporation by epithelial cells has been described in the course of experimental corneal healing [39].

\section{Therapeutic Role of AA}

Amino acids are important in several biological processes. They have been successfully used in the treatment of cardiological [46, 47], neurological [48] and endocrinological disorders [49]. In ophthalmology, they have been orally administered and commonly used to treat posterior segment diseases, often associated with nervous damage [50].

\section{Therapeutic Role of AA on the Ocular Surface}

A mixture containing the four AA described was orally administered to treat patients after photo refractive keratectomy (PRK), resulting in a statistically significant improvement of speed and quality of corneal repair [51, 52].

In DTS patients, the epithelial changes are characterized by an increase of the mean area of the opaque superficial cells [53]; this was demonstrated at confocal microscopy, and was related to the epithelial squamous metaplasia. The use of a mixture of AA was able to reduce the metaplasic
Fig. 2 Morphological processes in the corneal stroma and in the subbasal nerves in dry eye and after amino acids administration

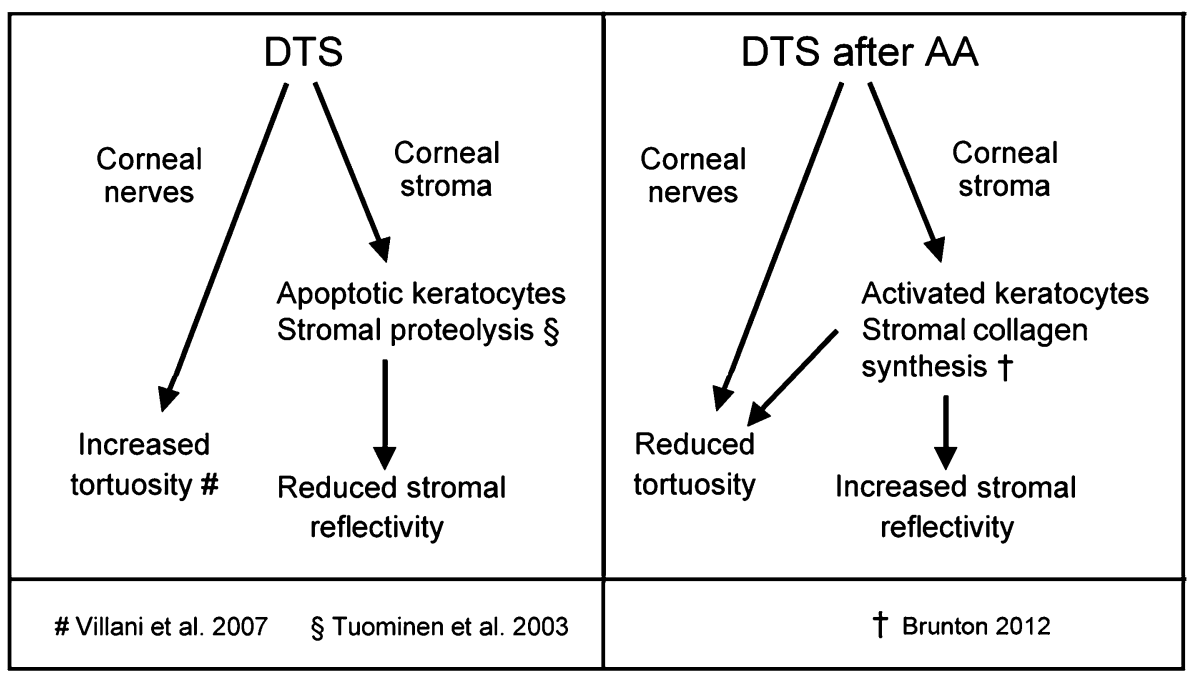


epithelial changes of the corneal epithelium. Another relevant aspect occurring in DTS patients are the changes in subbasal nerves morphology [54]. The treatment with AA was able to determine an improvement of the subbasal nerve pattern, and to statistically significantly ameliorate the slit lamp appearance of the corneal epithelium, after fluorescein stain. The stromal changes following the AA administration are illustrated in Fig. 2.

\section{Conclusions}

Substances deriving from food intake and nutraceutical products can influence the morphology and functions of the ocular surface system components. The tear film is a complex structure, whose organization must be balanced in order to accomplish the roles played in the protection of the ocular surface and in the maintenance of a good quality of vision. The tear film composition may depend on the availability of diet-derived substances, which may play a role as both precursors of useful molecules or regulators of the cellular functions in the secretory structures.

The knowledge about the effects and the properties of dietary components and supplements may help in recognizing possible ocular surface changes deriving from an unbalanced or deficient intake. On the other hand, it is possible to obtain favorable effects from the use of dietary supplements, which may be useful in the treatment of ocular surface diseases, such as dry eye.

Disclosure No potential conflicts of interest relevant to this article were reported.

\section{References}

Papers of particular interest, published recently, have been highlighted as:

- Of importance

- Of major importance

1. Gipson IK. The ocular surface: the challenge to enable and protect vision: the Friedenwald lecture. Invest Ophthalmol Vis Sci. 2007;48:4391-8.

2. - Green-Church KB, Butovich I, Willcox M, et al.: The international workshop on meibomian gland dysfunction: report of the subcommittee on tear film lipids and lipid-protein interactions in health and disease. Invest Ophthalmol Vis Sci 2011, 52:19791993. The work examines the meibomiam gland's contribution to tear film lipids and lipid-proteins interaction in health and disease.

3. Caffery BE. Influence of diet on tear function. Optom Vis Sci. 1991;68:58-72.

4. Guzman-Aranguez A, Argüeso P. Structure and biological roles of mucin-type O-glycans at the ocular surface. Ocul Surf. 2010;8:8-17.
5. Micali A, Puzzolo D, Pisani A, et al. Ultrastructural study of the conjunctival epithelium in the Mongolian gerbil (Meriones unguiculatus). Ophthalmic Res. 1998;30:244-54.

6. Unlisted authors: The definition and classification of dry eye disease: report of the Definition and Classification Subcommittee of the International Dry Eye WorkShop (2007). Ocul Surf 2007, 5:75-92.

7. • Dao AH, Spindle JD, Harp BA, et al.: Association of dyslipidemia in moderate to severe meibomian gland dysfunction. Am J Ophthalmol 2010, 150:371-375. This work demonstrates that patients with meibomian gland dysfunction have a higher incidence of elevated total cholesterol than the general population, with high serum LDL levels, and lower incidence of hypertriglyceridemia.

8. Aragona P, Giuffrida S, Di Stefano G, et al. Ocular surface changes in type 1 diabetic patients. Adv Exp Med Biol. 2002;506: 667-72.

9. Khandait DW, Vasudeo ND, Zodpey SP, et al. Vitamin A intake and xerophthalmia among Indian children. Public Health. 1999;113:69-72.

10. Eckert MJ, Perry JT, Sohn VY, et al. Incidence of low vitamin A levels and ocular symptoms after Roux-en-Y gastric bypass. Surg Obes Relat Dis. 2010;6:653-7.

11. Alwitry A. Vitamin A deficiency in coeliac disease. Br J Ophthalmol. 2000;84:1079-80.

12. Berthout A, Sellam M, Denimal F, et al. The eye and anorexia nervosa: a case report. J Fr Ophtalmol. 2007;30:e15.

13. Gaudiani JL, Braverman JM, Mascolo M, Mehler PS. Ophthalmic changes in severe anorexia nervosa: a case series. Int J Eat Disord. 2012;45:719-21.

14. Tovar AR, Gómez E, Bourges H, et al. Biochemical deficiency of pyridoxine does not affect interleukin-2 production of lymphocytes from patients with Sjögren's syndrome. Eur J Clin Nutr. 2002;56:1087-93.

15. Amemiya T. The eye and nutrition. Nihon Ganka Gakkai Zasshi. 1999;103:829-50.

16. $\bullet$ Roncone M, Bartlett H, Eperjesi F: Essential fatty acids for dry eye: A review. Cont Lens Anterior Eye 2010, 33:49-54. The work demonstrates supplementation with omega-3 may be beneficial in the treatment and the prevention of dry eye.

17. Rosenberg ES, Asbell PA. Essential fatty acids in the treatment of dry eye. Ocul Surf. 2010;8:18-28.

18. Russo GL. Dietary n-6 and n-3 polyunsaturated fatty acids: from biochemistry to clinical implications in cardiovascular prevention. Biochem Pharmacol. 2009;77:937-46.

19. Surette ME. The science behind dietary omega-3 fatty acids. Can Med Assoc J. 2008;178:177-80.

20. Simopoulos AP. Human requirement for N-3 polyunsaturated fatty acids. Poult Sci. 2000;79:961-70.

21. Willett WC. The role of dietary $n-6$ fatty acids in the prevention of cardiovascular disease. J Cardiovasc Med. 2007;8(Suppl 1):S42-5.

22. Miljanovic B, Trivedi KA, Dana MR, et al. Relation between dietary n-3 and n-6 fatty acids and clinically diagnosed dry eye syndrome in women. Am J Clin Nutr. 2005;82:887-93.

23. Aragona P, Bucolo C, Spinella R, et al. Systemic omega-6 essential fatty acid treatment and $\mathrm{PGE}_{1}$ tear content in Sjögren's syndrome patients. Invest Ophthalmol Vis Sci. 2005;46:4474-9.

24. Furse RK, Rossetti RG, Zurier RB. Gammalinolenic acid, an unsaturated fatty acid with anti-inflammatory properties, blocks amplification of IL-1 $\beta$ production by human monocytes. J Immunol. 2001;167:490-6.

25. Oxholm P, Asmussen K, Wiik A, Horrobin DF. Essential fatty acid status in cell membranes and plasma of patients with primary Sjögren's syndrome: correlations to clinical and immunologic variables using a new model for classification and assessment of disease manifestations. Prostaglandins Leukot Essent Fatty Acids. 1998;59:239-45. 
26. Macsai MS. The role of omega-3 dietary supplementation in blepharitis and meibomian gland dysfunction (an AOS thesis). Trans Am Ophthalmol Soc. 2008;106:336-56.

27. Erdinest N, Shmueli O, Grossman Y, et al. Anti-inflammatory effects of alpha linolenic acid on human corneal epithelial cells. Invest Ophthalmol Vis Sci. 2012;53:4396-406.

28. Serhan CN. Systems approach with inflammatory exudates uncovers novel anti-inflammatory and pro-resolving mediators. Prostaglandins Leukot Essent Fatty Acids. 2008;79:157-63.

29. Bazan NG, Eady TN, Khoutorova L, et al. Novel aspirin-triggered neuroprotectin D1 attenuates cerebral ischemic injury after experimental stroke. Exp Neurol. 2012;236:122-30.

30. Stark DT, Bazan NG. Neuroprotectin D1 induces neuronal survival and downregulation of amyloidogenic processing in Alzheimer's disease cellular models. Mol Neurobiol. 2011;43:131-8.

31. - He J, Bazan HE: Omega-3 fatty acids in dry eye and corneal nerve regeneration after refractive surgery. Prostaglandins Leukot Essent Fatty Acids 2010, 82:319-325. The study shows that topical application of omega-3 fatty acids and their derivatives resolvins are useful in treating dry eye and are able to prevent clinical complications such as corneal erosions and ulcerations.

32. Barabino S, Rolando M, Camicione P, et al. Systemic linoleic and gamma-linolenic acid therapy in dry eye syndrome with an inflammatory component. Cornea. 2003;22:97-101.

33. Creuzot C, Passemard M, Viau S, et al. Improvement of dry eye symptoms with polyunsaturated fatty acids. J Fr Ophtalmol. 2006;29:868-73.

34. Kokke KH, Morris JA, Lawrenson JG. Oral omega-6 essential fatty acid treatment in contact lens associated dry eye. Cont Lens Anterior Eye. 2008;31:141-6.

35. •• Brignole-Baudouin F, Baudouin C, Aragona P, et al.: A multicentre, double-masked, randomized, controlled trial assessing the effect of oral supplementation of omega- 3 and omega- 6 fatty acids on a conjunctival inflammatory marker in dry eye patients. Acta Ophthalmol 2011, 89:591-597. This trial demonstrates for the first time the anti-inflammatory effect of the supplementation of omega-3 in subjects suffering from dry eye.

36. $\bullet$ Meduri A, Aragona P, Grenga PL, Roszkowska AM: Effect of basic fibroblast growth factor on corneal epithelial healing after photorefractive keratectomy. J Refract Surg 2012, 28:220-223. This paper indicates that the remodeling of the corneal stroma may influence the normal development of the epithelium in subjects undergoing refractive surgery.

37. Micera A, Stampachiacchiere B, Mastrella L, et al. Effetto degli aminoacidi sulla sopravvivenza delle cellule epiteliali congiuntivali di linea. Ottica Fisiopatologica. 2008;3:1-8.

38. Chyun JH, Griminger P. Improvement of nitrogen retention by arginine and glycine supplementation and its relation to collagen synthesis in traumatized mature and aged rats. J Nutr. 1984;114: $1697-704$.
39. Zieske JD, Gipson IK. Protein synthesis during corneal epithelial wound healing. Invest Ophthalmol Vis Sci. 1986;27:1-7.

40. Barbul A. Proline precursors to sustain mammalian collagen synthesis. J Nutr. 2008;138:2021S-4S.

41. Ottani V, Martini D, Franchi M, et al. Hierarchical structures in fibrillar collagens. Micron. 2002;33:587-96.

42. Wu G, Bazer FW, Burghardt RC, et al. Proline and hydroxyproline metabolism: implications for animal and human nutrition. Amino Acids. 2011;40:1053-63.

43. Brunton JA, Baldwin MP, Hanna R, Bertolo RF. Proline supplementation to parenteral nutrition results in greater rates of protein synthesis in the muscle, skin, and small intestine in neonatal Yucatan miniature piglets. J Nutr. 2012;142:1004-8.

44. Yamauchi M, Sricholpech M. Lysine post-translational modifications of collagen. Essays Biochem. 2012;52:113-33.

45. Mohan RR, Tovey JCK, Gupta R, et al. Decorin biology, expression, function and therapy in the cornea. Curr Mol Med. 2011;11:110-28.

46. Aquilani R, Viglio S, Iadarola P, et al. Oral amino acid supplements improve exercise capacities in elderly patients with chronic heart failure. Am J Cardiol. 2008;101:104-10.

47. Scognamiglio $\mathrm{R}$, Testa A, Aquilani $\mathrm{R}$, et al. Impairment in walking capacity and myocardial function in the elderly: is there a role for nonpharmacologic therapy with nutritional amino acid supplements? Am J Cardiol. 2008;101:78-81.

48. Aquilani R, Boselli M, Boschi F, et al. Branched-chain amino acids may improve recovery from a vegetative or minimally conscious state in patients with traumatic brain injury: a pilot study. Arch Phys Med Rehabil. 2008;89:1642-7.

49. Aquilani R. Oral amino acid administration in patients with diabetes mellitus: supplementation or metabolic therapy? Am J Cardiol. 2004;93:21-2.

50. Karlsson JO, Sjöstrand J. Axonal transport of proteins in retinal ganglion cells. Aminoacid incorporation into rapidly transported proteins and distribution of radioactivity to the lateral geniculate body and superior colliculus. Brain Res. 1972;37:279-85.

51. Vinciguerra P, Camesasca FI, Ponzin D. Use of amino acids in refractive surgery. J Refract Surg. 2002;18:S374-7.

52. Torres Munoz I, Grizzi F, Russo C, et al. The role of amino acids in corneal stromal healing: a method for evaluating cellular density and extracellular matrix distribution. J Refract Surg. 2003;19:S227-30.

53. Chen JJ, Rao K, Pflugfelder SC. Corneal epithelial opacity in dysfunctional tear syndrome. Am J Ophthalmol. 2009;148: 376-82.

54. Tuominen IS, Konttinen YT, Vesaluoma MH, et al. Corneal innervation and morphology in primary Sjögren's syndrome. Invest Ophthalmol Vis Sci. 2003;44:2545-9. 